\title{
Anti-Inflammatory Effects of Hexane Fraction from White Rose Flower Extracts via Inhibition of Inflammatory Repertoires
}

\author{
Hwa-Jeong Lee ${ }^{1}$, Han-Seok Kim² ${ }^{2}$, Seung Tae Kim ${ }^{5}$, Dongsun Park ${ }^{4}$, Jin Tae Hong ${ }^{3}$, Yun-Bae Kim ${ }^{4, *}$ and \\ Seong Soo Joo ${ }^{5, *}$ \\ ${ }^{1}$ Department of Beauty Design, Dongshin University, Naju 520-714, \\ ${ }^{2}$ Department of Cosmeteology, Chung Am University, Suncheon 540-743, \\ ${ }^{3}$ College of Pharmacy, Chungbuk National University, Cheongju 361-763, \\ ${ }^{4}$ College of Veterinary Medicine and Research Institute of Veterinary Medicine, Chungbuk National University, Cheongju 361-763, \\ ${ }^{5}$ Department of Marine Molecular Biotechnology, College of Life Science, Gangneung-Wonju National University, Wonju 210-702, \\ Republic of Korea
}

\begin{abstract}
In this study, we determined the anti-inflammatory activity and mechanism of action of a hexane fraction (hWRF) obtained from white Rosa hybrida flowers by employing various assays such as quantitative real-time PCR, Western blotting, and Electrophoretic-Mobility Shift Assay (EMSA). The results revealed that the hWRF had excellent anti-inflammatory potency by reducing inflammatory repertoires, such as inducible nitric oxide synthase (iNOS), interleukin-1 $\beta$, and cyclooxygenase-2 (COX-2) in RAW264.7 cells when stimulated with lipopolysaccharide (LPS), a pro-inflammatory mediator. The reduction of nitric oxide (NO) release from RAW 264.7 cells supported the anti-inflammatory effect of hWRF. Interestingly, hWRF effectively inhibited LPS-mediated nuclear

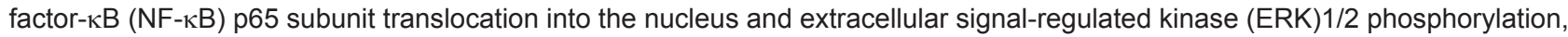
suggesting that hWRF anti-inflammatory activity may be based on inhibition of the NF-kB and MAPK pathways. Based on the findings described in this study, hWRF holds promise for use as a potential anti-inflammatory agent for either therapeutic or functional adjuvant purposes.
\end{abstract}

Key Words: Rosa hybrida, Inflammatory repertoire, RAW 264.7, Lipopolysaccharide, Nuclear factor-кB

\section{INTRODUCTION}

For decades, many studies have shown that pharmacologically active compounds are present in various plants and can act as special metabolites that allow plants to interact with factors found in its environment, such as plant-insect, plantmicroorganism and plant-plant interactions (Dixon, 2001; Harborne, 2001; Argolo et al., 2004). Among the several small molecules retained in plants, polyphenols (hydrolysabletannins/phenylpropanoids) are the most abundant antioxidants in our diet and have been shown to play a possible role in the prevention several chronic diseases (Bravo, 1998; Hotta et al., 2002; Gosslau et al., 2011b). Polyphenol antioxidants, such as resveratrol, inhibit the occurrence and/or growth of mammalian tumors (Jang et al., 2008), and a wide variety of phenolic substances derived from plants have been shown to display marked antioxidant and anti-inflammatory activities, which contribute to their chemopreventive potential (Surh et al., 2001). Moreover, natural anti-inflammatory or immunomodulatory plant metabolites, which are abundant in fractionated extracts, have therapeutic effects against various chronic diseases, by inducing or suppressing specific cellular inflammatory activities and the associated molecular signaling pathways (Aravindaram and Yang, 2010). Some polyphenols, such as catechines from green tea, appear to be the primary bioactive compounds in the suppression of key cytokines that are secreted during the inflammation cascade (De Mjia et al., 2009; Gosslau et al., 2011a).

The rose (genus Rosa), allied to its use as a source of aromatic oils for the perfume industry, has been an alternative model for pharmaceutical candidates to treat diabetes mellitus, pain, and inflammatory diseases (Cho et al., 2003; $\mathrm{Ng}$ et al., 2005). Previously, we reported that the hexane fraction from white rose flowers, which may contain high amounts of

Received May 25, 2011 Revised Jun 28, 2011 Accepted Jun 29, 2011

*Corresponding Authors

E-mail: ssj66@gwnu.ac.kr (Joo SS), solar93@cbu.ac.kr (Kim YB) Tel: +82-33-640-2856 (Joo SS), +82-43-261-3358 (Kim YB) Fax: +82-33-640-2340 (Joo SS), +82-43-271-3246 (Kim YB) 
gallic acid and volatiles (1-butanol, dodecyl acrylate and cyclododecane), displayed a broad range of anti-oxidant, antimicrobial and anti-allergic effects (Jeon et al., 2009; Park et al., 2009; Joo et al., 2010). In the present study, we examined the anti-inflammatory properties of the hexane fraction from the white rose flower, which may be used as a natural source of bioactive compounds.

\section{MATERIALS AND METHODS}

\section{Preparation of hexane fraction (hWRF)}

Finely ground flower powders $(410 \mathrm{~g})$ were extracted with methanol at room temperature for $24 \mathrm{~h}$. The ratio of flower powder to solvent was 1:20 (w/v). The resulting slurries were filtered through Whatman no. 1 filter paper. This procedure was repeated twice for the residue, and the filtrates were combined. All of the filtrates were collected and concentrated under vacuum at $50^{\circ} \mathrm{C}$ and freeze-dried ( $54 \%$ yield). The freezedried methanol extract $(1 \mathrm{~g})$ was then redissolved in methanol $(1.5 \mathrm{ml})$, and $20 \mathrm{ml}$ of water was added (methanol/water, $1.5: 20, \mathrm{v} / \mathrm{v})$. This mixture was then sequentially partitioned with hexane and butanol (same solvent ratio as water). Three resulting fractions $\left(\mathrm{H}_{2} \mathrm{O}\right.$, butanol, and hexane layer) were obtained, evaporated to dryness under vacuum, and used directly in the anti-inflammatory tests. The hexene fraction was dissolved in $10 \%$ DMSO, and stock solutions were prepared at high concentrations so that the final DMSO concentration was not higher than $0.1 \%$ during all experiments.

\section{Cell culture}

Raw 264.7 (Mouse leukaemic monocyte macrophage cell line) cells were grown in Dulbecco's Modified Eagle's Medium (DMEM) (Hyclone, Logan, UT, USA) supplemented with 10\% FBS, $4 \mathrm{mM} / \mathrm{L}$ L-glutamate (Invitrogen, Carlsbad CA, USA), $100 \mathrm{U} / \mathrm{ml}$ penicillin and $100 \mu \mathrm{g} / \mathrm{ml}$ streptomycin (Invitrogen). Cultures were maintained under $5 \% \mathrm{CO}_{2}$ at $37^{\circ} \mathrm{C}$ in tissue culture flasks. For all experiments, the cells were grown to greater than $90 \%$ confluency and subjected to no more than 20 cell passages.

\section{Cellular Cytotoxicity (lactate dehydrogenase) assay}

The cytotoxicity induced by hWRF was quantified by measuring lactate dehydrogenase (LDH) release by RAW 264.7 cells. The LDH content was determined using a commercial non-radioactive LDH assay kit (Promega, Madison, WI, USA), which is based on a coupled enzymatic reaction that converts tetrazolium salt into a red formazan product. The increase in the amount of formazan produced in the culture supernatant directly correlates with the increase in the number of lysed cells. The formazan was quantified spectrophotometrically by measuring the absorbance at $490 \mathrm{~nm}$ (Molecular Devices, Sunnyvale, CA, USA).

\section{Nitric oxide (NO) production in RWA 264.7}

RAW 264.7 cells $\left(1 \times 10^{6}\right.$ cells $\left./ \mathrm{ml}\right)$ were seeded on a $24-$ well tissue culture plate and pre-incubated at $37^{\circ} \mathrm{C}$ for $12 \mathrm{~h}$ to achieve stable attachment. The wells were then washed with phosphate buffered saline (PBS) after pre-incubation, refreshed with $1 \%$ FBS DMEM containing the samples (lipopolysaccharide (LPS), ascorbic acid (Asc), and hWRF (0.1 to $10 \mu \mathrm{g} / \mathrm{ml})$ ) and incubated for $24 \mathrm{~h}$. NO production was then monitored by measuring nitrite levels in the culture media using the Griess reagent (Promega).

\section{Quantitative real-time polymerase chain reaction (PCR)}

Total RNA extracts from RAW 264.7 cells were stimulated by LPS, which was prepared by the Trizol method (Invitrogen). cDNA was synthesized from RNA by the reverse transcription of $1 \mu \mathrm{g}$ of total RNA using the Improm-Il reverse transcription system (Promega) and oligo dT primers in a total volume of $20 \mu \mathrm{l}$. PCR amplification was performed using the primers shown in Table 1 (Bioneer, Deajeon, Korea). Quantitative realtime PCR reactions were run on a Rotor-Gene 6000 (Corbett Research, Sydney, Australia) using SYBR Green PCR Master Mix (Qiagen, Valencia, CA, USA) in $20 \mu$ reaction mixtures. Each real-time-PCR master mix contained $10 \mu \mathrm{l} 2 \mathrm{X}$ enzyme mastermix, $7.0 \mu \mathrm{l}$ RNase free water, $1 \mu \mathrm{l}$ of each primer (10 $\mathrm{pM}$ each) and $1 \mu \mathrm{l}$ diluted template. PCR was performed with an initial pre-incubation step for $10 \mathrm{~min}$ at $95^{\circ} \mathrm{C}$, followed by 45 cycles of $95^{\circ} \mathrm{C}$ for $15 \mathrm{~s}$, annealing at $52^{\circ} \mathrm{C}$ for $15 \mathrm{~s}$ and extension at $72^{\circ} \mathrm{C}$ for $10 \mathrm{~s}$. Melting curve analysis was used to confirm formation of the expected PCR product, and products from all assays were subjected to $1.2 \%$ agarose gel electrophoresis to confirm that they had the correct lengths. An interrun calibrator was used, and a standard curve was created for each gene to obtain PCR efficiencies. Relative sample expression levels were calculated using Rotor-Gene 6000 Series Software 1.7, and were expressed relative to $\beta$-actin and corrected for between run variability. Data for the experimental samples were expressed as the percentage of the internal control gene.

Table 1. Primer sequences used for real-time PCR

\begin{tabular}{|c|c|c|c|c|}
\hline Gene (murine) & Primer & Nucleotide sequences & $\begin{array}{l}\text { Product } \\
\text { size (bp) }\end{array}$ & Accession no. \\
\hline \multirow[t]{2}{*}{ iNOS } & 5' Primer & 5'-TGCCCCTGGAAGTTTCTCTT & 252 & NM_010927 \\
\hline & 3' Primer & 5'-ACTGCCCCAGTTTTTGATCC & & \\
\hline \multirow[t]{2}{*}{$\mathrm{IL}-1 \beta$} & 5' Primer & 5'-AGCTGTGGCAGCTACCTGTG & 522 & NM_008361 \\
\hline & 3' Primer & 5'-GCTCTGCTTGTGAGGTGCTG & & \\
\hline \multirow[t]{2}{*}{$\operatorname{cox}-2$} & 5' Primer & 5'-CTGACCCACTTCAAGGGAGTCT & 476 & NM_011198 \\
\hline & 3' Primer & 5'-CCATCCTTGAAAAGGCGCAGTT & & \\
\hline \multirow[t]{2}{*}{$\beta$-actin } & 5' Primer & 5'-CTAGGCACCAGGGTGTGATG & 291 & NM_007393 \\
\hline & 3' Primer & 5'-CTACGTACATGGCTGGGGTG & & \\
\hline
\end{tabular}




\section{Electrophoretic-mobility shift (EMSA) assay}

Nuclear extracts for EMSA were prepared from RAW 264.7 cells using Nuclear Extraction Reagents (Pierce, IL, USA) according to the manufacturer's instructions. EMSA was performed using the Panomics EMSA kit (Panomics, Fremont, CA, USA). In brief, nuclear extracts containing equal amounts of proteins for each sample were incubated with poly (dl-dC) $(1 \mu \mathrm{g} / \mu \mathrm{l})$ for $5 \mathrm{~min}$, followed by the addition of binding buffer (20 mM HEPES pH 7.9, 1 mM DTT, 0.1 mM EDTA, 50 mM $\mathrm{KCl}, 5 \%$ glycerol and $200 \mu \mathrm{g} / \mathrm{ml} \mathrm{BSA}$ ) and biotinylated oligo $(10 \mathrm{ng} / \mu \mathrm{l})$. To control for the specificity of binding for selected samples, a 5-fold excess of unlabeled oligo was added prior to the addition of the biotinylated probe. Binding reaction mixtures were incubated for $30 \mathrm{~min}$ at room temperature. ProteinDNA complexes were separated on $6 \%$ non-denaturing polyacrylamide gel in $0.5 \mathrm{X}$ Tris-borate/EDTA buffer $(0.1 \mathrm{M}$ Tris, $0.09 \mathrm{M}$ boric acid containing $1 \mathrm{mM}$ EDTA) at $4^{\circ} \mathrm{C}$. After electrophoresis, the gels were transferred to a positively charged nylon transfer membrane (Amersham Bioscience, Piscataway NJ, USA). Transferred oligos were immobilized by UV crosslinking for $10 \mathrm{~min}$. To detect bound oligos, membranes were blocked using blocking buffer (Panomics EMSA Gel-Shift Kit). Streptavidin-HRPO was then added and blots were developed by ECL according to the manufacturer's instructions (Amersham Bioscience).

\section{Western blot analysis}

RAW 264.7 cells were lysed in 1\% RIPA buffer containing protease and phosphatase inhibitors (Roche, Mannheim, Germany) and whole cell lysates were separated by $10 \%$ SDSPAGE. After electrophoresis, proteins were transferred onto polyvinylidene fluoride (PVDF) membranes and the membranes were blocked with $5 \%$ skim milk in Tris-buffered saline solution containg $0.1 \%$ Tween-20. The membrane was then immunoblotted with primary antibodies, anti-iNOS, antiextracelullar signal-regulated protein kinase, anti-phosphoERK and anti-Glyceraldehyde 3-phosphate dehydrogenase (GAPDH) (Santa Cruz Biotechnology, CA, USA), which was followed by incubation with horseradish peroxidase-conjugated anti-rabbit or anti-mouse secondary antibodies (Stressgen, CA, USA). Blots were developed using an ECL solution. Densitometric measurements were conduction using the Gelquant software (MiniBIS Pro, Jerusalem, Israel).

\section{Statistical analysis}

The statistical analyses used to determine the differences between the groups included a one-way ANOVA with a Dunnet's post-hoc test, which was performed using the SPSS software (v. 13). Statistical significance was set a priori at $p<0.05$.

\section{RESULTS}

To investigate the cytotoxicity of hWRF, RAW 264.7 cells were incubated with various concentrations of hWRF (0.01 to $100 \mu \mathrm{g} / \mathrm{ml})$. As shown in Fig. 1, hWRF was not cytotoxic up to 24 hours at concentrations lower than $10 \mu \mathrm{g} / \mathrm{ml}$; however, a significant increase in LDH release from the cells was observed at higher concentrations $(100 \mu \mathrm{g} / \mathrm{ml})$, indicating that these higher concentrations were cytotoxic. Because the cytotoxicity of hWRF at $100 \mu \mathrm{g} / \mathrm{ml}$ was so large, higher concentrations were not tested. Additionally, to stimulate NO production

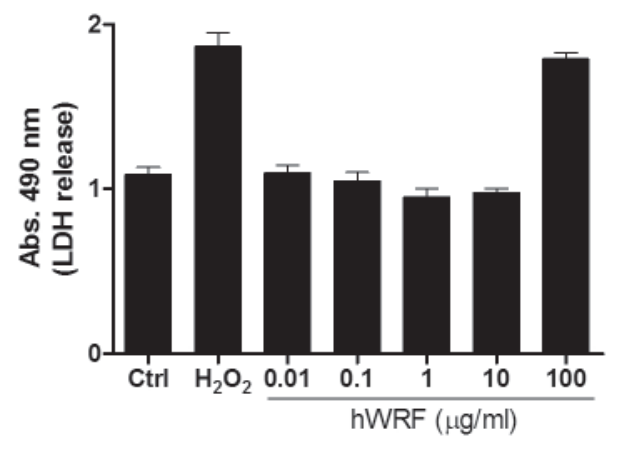

Fig. 1. Cytotoxicity in RAW 264.7 cells. RAW 264.7 cells were exposed to different concentrations of hWRF $(0.01$ to $100 \mu \mathrm{g} / \mathrm{ml})$ for the indicated times. Concentration dependent cytotoxicity was measured based on the level of LDH release into the culture supernatant.

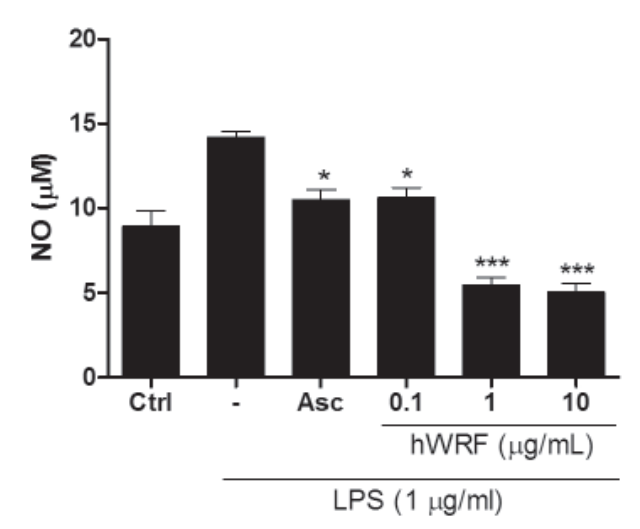

Fig. 2. Inhibition of nitric oxide in RAW 264.7 cells. Cells were seeded on a 96-well culture plate, treated with hWRF $(0.1$ to $10 \mu \mathrm{g} /$ $\mathrm{ml})$, and incubated for $24 \mathrm{~h}$. Ascorbic acid $(100 \mu \mathrm{M})$ was used as a positive control, and LPS $(1 \mu \mathrm{g} / \mathrm{ml})$ was used as a cell stimulator. The presence of NO was detected using the Griess reagent and measured at $540 \mathrm{~nm}$. The experiments were performed in triplicate and the results are expressed as the mean \pm SD. ${ }^{*} p<0.05,{ }^{* * *} p<$ 0.001 compared with positive control, LPS.

in RAW 267.4 cells, $1 \mu \mathrm{g} / \mathrm{ml}$ LPS was added and varying concentrations of the cells were co-treated with hWRF (0.1 to 10 $\mu \mathrm{g} / \mathrm{ml}$ ) for up to $24 \mathrm{~h}$. As shown in Fig. 2, hWRF significantly inhibited NO production at concentrations ranging from 1 to 10 $\mu \mathrm{g} / \mathrm{ml}(p<0.001)$, which was more effective than ascorbic acid $(p<0.01)$. Fig. 3 clearly demonstrates that hWRF can effectively control the expression of those pro-inflammatory mediators and repertoires, suggesting that hWRF can down-regulate LPS-induced iNOS, IL-1 $\beta$ and COX-2 expression at the transcriptional level. To determine whether the anti-inflammatory activities of hWRF are mediated through the MAP kinase pathway, LPS-induced phosphorylation of ERK was analyzed. In addition, the protein levels of iNOS were also analyzed by immunoblotting. As shown in Fig. 4A, phosphoylated ERK was effectively attenuated, and iNOS were coincidently reduced in the presence of hWRF. To confirm that hWRF might be correlated with the inhibition of $\mathrm{NF}-\mathrm{kB}$ activation, we examined translocation of NF-kB p65 subumit into the nucleus, which binds to specific DNA sequences. Fig. 4B revealed that hWRF effectively inhibited binding to specific NF-kB p65 DNA se- 

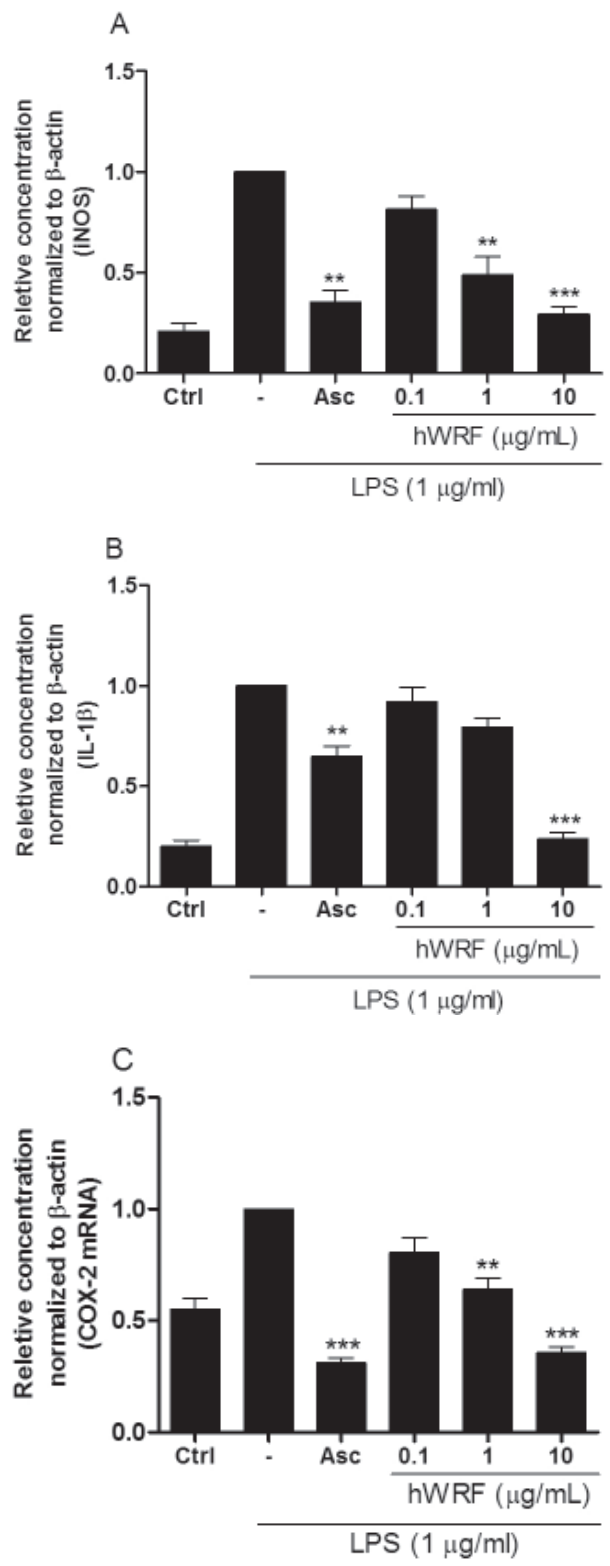

Fig. 3. Inflammatory cytokine mRNA expression in RAW264.7 cells. Cells were cultured for 24 hours as described in the Methods and Materials, and Asc was used as a positive control for mRNA analysis. The mRNA levels of iNOS, IL-1 $\beta$, COX-2, and $\beta$-actin were analyzed by real-time RT-PCR. Results were internally confirmed by comparative cycle count ( $\mathrm{Ct}$, cycle number threshold) against $\beta$-actin, which was used as the standard gene. The LPSstimulated group was used for a relative comparison of each group. Results are mean \pm S.D. $(n=3) .{ }^{* *} p<0.01,{ }^{* * *} p<0.001$. Asc: ascorbic acid.

quences, which strongly suggests that hWRF effectively attenuated LPS-induced expression of pro-inflammatory proteins.

\section{DISCUSSION}

In our previous study, we determined that the hexane fraction of the white rose flower extract contained various
A

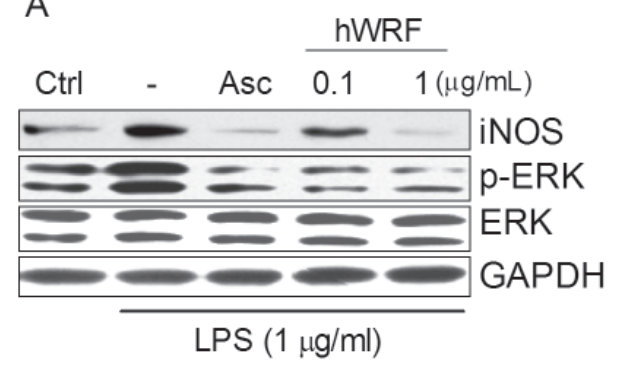

B

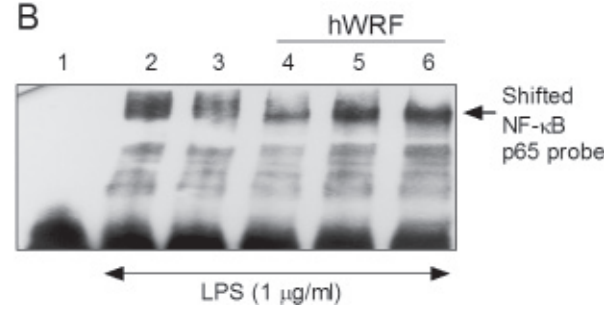

Fig. 4. Electrophoretic-mobility Shift Assay for NF-kB 65 and Western blot profiles of inflammatory-associated proteins and phosphorylated ERK. (A) LPS-stimulated RAW 264.7 cells were treated with hWRF or Asc for $60 \mathrm{~min}$, followed by preparation of nuclear extracts for EMSA as described in Materials and Methods. Lane 1, labeled EMSA probe only with no sample. Lane 2, labeled EMSA probe with LPS-treated sample. Lanes 3, labeled EMSA probe with $100 \mu \mathrm{M}$ Asc. Lane 4 to 6 , labeled EMSA probe with 1, 0.1 , or $0.01 \mu \mathrm{g}$ hWRF. Lane 2 to 6 was stimulated with LPS $1 \mu \mathrm{g}$. (B) Analysis of iNOS, phosphor-ERK and ERK in lysates of RAW 264.7 cells. Asc was used as a positive control.

Table 2. Volatile components identified by GC-MS in hexane fraction

\begin{tabular}{lcc}
\hline \multicolumn{1}{c}{ Compound } & Retention time $(\mathrm{min})$ & Content $(\%)$ \\
\hline 1-Butanol & 7.17 & 7.52 \\
Dodecane & 9.10 & 2.14 \\
Tetradecane & 17.37 & 1.71 \\
Benzene & 18.36 & 1.25 \\
Hexadecane & 25.45 & 0.87 \\
Cyclododecane & 38.54 & 23.83 \\
Dodecyl acrylate & 39.32 & 41.17 \\
Ethanone & 49.56 & 2.61 \\
\hline
\end{tabular}

functional volatiles (Table 2) and the abundant amount of total polyphenols $(330.3 \pm 20.1 \mathrm{mg}$ of gallic acid equival $/ \mathrm{g}$ of dry weight), which was approximately 2 -fold higher than the amount observed in the butanol fraction and 2.6-fold higher than the amount observed in the water fraction (Park et al., 2009; Joo et al., 2010). These results suggested that the hexane fraction from white rose flower extracts may have a more potent functional activity and, thus, was selected for more indepth anti-inflammatory analysis (Joo et al., 2010).

The primary anti-inflammatory evidence was shown in NO inhibition assay in RAW 264.7 cells, suggesting that hWRF may control NO concentration at the cellular level by inhibiting $\mathrm{NO}$ production (Fig. 2). $\mathrm{NO}$ and prostaglandin $\mathrm{E}_{2}$ have been shown to be closely involved in the development of inflammation, and these two inflammatory mediators are associated with the expression iNOS and COX-2 (Sautebin, 2000). Moreover, IL-1 $\beta$ is also a well-known pro-inflammatory cytokine. 
Results revealed that hWRF can effectively control the expression of those pro-inflammatory mediators and repertoires, suggesting that hWRF can down-regulate LPS-induced iNOS, IL-1 $\beta$ and COX-2 expression at the transcriptional level (Fig. 3, 4) (Warner et al., 1995). These results were confirmed by the attenuation of ERK phosphorylation and a coincident reduction of iNOS when treated with hWRF (Fig. 4A). The NF-kB transcription factor has been shown to play an important role in LPS-induced expression of pro-inflammatory proteins (Xie et al., 1994; Nagano et al., 1998; Lee, 2011). Thus, to investigate the molecular mechanism of hWRF-mediated inhibition of pro-inflammatory repertoires, such as iNOS and IL-1 $\beta$, NF$\kappa B$ transcription activity was investigated using immunoblot analysis of NF-kB p65 with the EMSA supershift assay (Fig. 4B).

At the molecular level, hWRF appreciably controlled the expression of inflammatory repertoires by inhibiting the activation of NF-kB, which can induce the overexpression of proinflammatory cytokine genes in the nucleus, thereby effectively attenuate LPS-induced expression of pro-inflammatory proteins. Since inhibition of NF-kB has been reported to be an effective approach for the treatment of inflammatory disorders (Karin et al., 2004), hWRF, which interfered with NF-kB signal transduction, may be a good therapeutic candidate for treatment of inflammation. In turn, hWRF may inhibit iNOS, COX-2 expression and the pro-inflammatory related genes by preventing the nuclear translocation of the p65subunit of NF$\kappa B$. Since macrophages play a central role in the host's defense against bacterial infection due to their phagocytic, cytotoxic, and intracellular killing capacities (Adams and Hamilton, 1984), hWRF may be used for the treatment of a broad range of inflammatory disorders, including topical inflammation and worsening chronic dermatitis, such as atopic dermatitis (Jeon et al., 2009).

In conclusion, our results suggest that hWRF may be a favorable candidate for treatment of inflammatory disorders, and the anti-inflammatory activity of hWRF may be a function of the inhibition of iNOS and COX-2 expression via inactivation with NF-kB. Therefore, hWRF, a natural product, may serve as safe and effective anti-inflammatory agent, which could be used in functional food or for therapeutic purposes.

\section{ACKNOWLEDGMENTS}

This research was supported by the Dongshin University research grants.

\section{REFERENCES}

Adams, D. O. and Hamilton, T. A. (1984) The cell biology of macrophage activation. Annu. Rev. Immunol. 2, 283-318.

Aravindaram, K. and Yang, N. S. (2010) Anti-inflammatory plant natural products for cancer therapy. Planta Med. 76, 1103-1117.

Argolo, A. C. C., Sant'Ana, A. E. G., Pletsch, M. and Coelho, L. C. B. B.
(2004) Antioxidant activity of leaf extracts from Bauhinia monandra. Bioresour. Technol. 95, 229-233.

Bravo, L. (1998) Polyphenols: chemistry, dietary sources, metabolism, and nutritional significance. Nutr. Rev. 56, 317-333.

Cho, E. J., Yokozawa, T., Rhyu, D. Y., Kim, S. L., Shibahara, N. and Park, J. C. (2003). Study on the inhibitory effects of Korean medicinal plants and their main compounds on the 1,1-diphenyl-2picrylhydrazyl radical. Phytomedicine 10, 544-551.

De Mjia, E. G., Ramirez-Mares, M. V. and Puangpraphant S. (2009) Bioactive components of tea: cancer, inflammation and behavior. Brain Behav. Immun. 23, 721-731.

Dixon, R. A. (2001) Natural products and plant disease resistance. Nature 411, 843-847.

Harborne, J. B. (2001) Twenty-five years of ecology. Nat. Prod. Rep. 18, 361-379.

Hotta, H., Nagano, S., Ueda, M., Tsujino, Y., Koyama, J. and Osakai, T. (2002) Higher radical scavenging activities of polyphenolic antioxidants can be ascribed to chemical reactions following their oxidation. Biochim. Biophys. Acta 1572, 123-132.

Gosslau, A., En Jao, D. L., Huang, M. T., Ho, C. T., Evans, D., Rawson, N. E. and Chen, K. Y. (2011a) Effects of the black tea polyphenol theaflavin-2 on apoptotic and inflammatory pathways in vitro and in vivo. Food Res. 55, 198-208.

Gosslau, A., Li, S., Ho, C. T., Chen, K. Y. and Rawson, N. E. (2011b) The importance of natural product characterization in studies of their anti-inflammatory activity. Mol. Nutr. Food Res. 55, 74-82.

Jang, H. W., Ka, M. H. and Lee K. G. (2008) Antioxidant activity and characterization of volatile extracts of Capsicum annuum L. and Allium spp. Flavour. Fragr. J. 23, 178-184.

Jeon, J. H., Kwon, S. C., Park, D. S., Shin, S., Jeong, J. H., Park, S. Y Hwang, S. Y., Kim, Y. B. and Joo, S. S. (2009) Anti-allergic effects of white rose pertal extract and anti-atopic properties of its hexae fraction. Arch. Pharm. Res. 32, 823-830.

Joo, S. S., Kim, Y. B. and Lee, D. I. (2010) Antimicrobial and antioxidant properties of secondary metabolites from white rose flower. Plant Pathol. J. 26, 57-62.

Karin, M., Yamamoto, Y. and Wang, Q. M. (2004) The IKK NFkappa B system: a treasure trove for drug development. Nat. Rev. Drug Discov. 3, 17-26.

Lee, J. K. (2011) Anti-inflammatory effects of eriodictyol in lipopolysaccharide-stimulated Raw264.7 murine macrophages. Arch. Pharm. Res. 34, 671-679.

Nagano, H., Shindo, M., Sakon, S., Nisinaka, S., Mihara, M., Yagita, H. and Okumura, K. (1998) Differential regulation of IkappaB kinase alpha and beta by two upstream kinases NF-kappaB-inducing kinase and mitogen-activated protein kinase/ERK kinase kinase-1. Proc Natl Acad Si USA 95, 3537-3542.

Ng, T. B., Gao, W., Li, L., Niu, S. M., Zhao, L., Liu, J., Shi, L. S., Fu, M., and Liu, F. (2005) Rose (Rosa rugosa)-flower extract increases the activities of antioxidant enzymes and their gene expression and reduces lipid peroxidation. Biochem. Cell Biol. 83, 78-85.

Park, D. S., Jeon, J. H., Kwon, S. C., Shin, S., Jang, J. Y., Jeong, H. S., Lee, D. I., Kim, Y. B. and Joo SS. (2009) Antioxidative activities of white rose flower extract and pharmaceutical advantages. Biochem. Cell Biol. 87, 943-952.

Sautebin, L. (2000) Prostaglandins and nitric oxide as molecular targets for anti-inflammatory therapy. Fitoterapia 71, S48-57.

Surh, Y. J., Na, H. K., Lee, J. Y. and Keum, Y. S. (2001) Molecular mechanisms underlying anti-tumor promoting activities of heatprocessed Panax ginseng C.A. Meyer. J. Korean Med. Sci. 16, S38-41.

Warner, R. L., Paine, R. 3rd., Christensen, P. J., Marletta, M. A., Richards, M. K., Wilcoxen, S. E. and Ward, P. A. (1995) Lung sources and cytokine requirements for in vivo expression of inducible nitric oxide synthase. Am. J. Respir. Cell Mol. Biol. 12, 649-661.

Xie, Q. W., Kashiwabara, Y. and Nathan, C. (1994) Role of transcription factor NF-kappa B/Rel in induction of nitric oxide synthase. J. Biol. Chem. 269, 4705-4708. 